\title{
EDGE FINITE ELEMENTS FOR THE APPROXIMATION OF MAXWELL RESOLVENT OPERATOR
}

\author{
Daniele Boffi ${ }^{1}$ AND Lucia Gastaldi ${ }^{2}$
}

\begin{abstract}
In this paper we consider the Maxwell resolvent operator and its finite element approximation. In this framework it is natural the use of the edge element spaces and to impose the divergence constraint in a weak sense with the introduction of a Lagrange multiplier, following an idea by Kikuchi [14]. We shall review some of the known properties for edge element approximations and prove some new result. In particular we shall prove a uniform convergence in the $L^{2}$ norm for the sequence of discrete operators. These results, together with a general theory introduced by Brezzi, Rappaz and Raviart [8], allow an immediate proof of convergence for the finite element approximation of the time-harmonic Maxwell system.
\end{abstract}

Mathematics Subject Classification. 65N25, 65N30.

Received: September 7, 2001. Revised: January 23, 2002.

\section{INTRODUCTION}

In the last few years the interest of the mathematical community in the Maxwell system has increased and important results have been obtained about its numerical approximation. In particular several papers consider the approximation of Maxwell interior eigenvalues (see, for instance, $[3,6,9,14,17]$ ) and the related problem of the approximation of the time-harmonic Maxwell equations (see $[11,16]$ ). In this framework the edge finite elements are the natural choice for getting performant schemes. In general the use of edge elements is the main way in order to achieve convergence and optimal accuracy. A common tool for the edge element analysis of the above mentioned models is to show a suitable convergence property of the discrete operators sequence. This is the case, for instance, of the properties related to the Fortin operator [3-5], or to a discrete compactness [15,17].

The aim of this paper is to enlighten several theoretical properties of edge element spaces. In particular, a more general convergence of the discrete operators sequence will be proven. Our proof relies on the theory presented in [12] and [3,5], together with a suitable modification of the techniques presented in [2]. The main result requires minimal regularity hypotheses, and in particular standard shape-regularity for the mesh sequences (no quasiuniformity is required). The regularity assumption (6) could be further weakened by making use of recent results by Schöberl (see Rem. 1).

\footnotetext{
Keywords and phrases. Edge finite elements, time-harmonic Maxwell's equations, mixed finite elements.

1 Dipartimento di Matematica, Università di Pavia, 27100 Pavia, Italy. e-mail: boffi@dimat.unipv.it

2 Dipartimento di Matematica, Università di Brescia, 25133 Brescia, Italy. e-mail: gastaldi@bsing.ing.unibs.it
} 
As an application of our results, we shall consider the approximation of the time-harmonic Maxwell equations. Given $\omega$ suitably chosen in $\mathbb{R}$ and $\underline{F}: \Omega \rightarrow \mathbb{C}^{3}$ with $\operatorname{div} \underline{F}=0$ in $\Omega$, we look for $\underline{u}: \Omega \rightarrow \mathbb{C}^{3}$ such that

$$
\begin{array}{ll}
\operatorname{curl}\left(\mu^{-1} \operatorname{curl} \underline{u}\right)-\left(\omega^{2} \varepsilon-j \omega \sigma\right) \underline{u}=-j \omega \underline{F} & \text { in } \Omega, \\
\operatorname{div}((j \omega \varepsilon+\sigma) \underline{u})=0 & \text { in } \Omega, \\
\underline{u} \times \underline{n}=0 & \text { on } \partial \Omega .
\end{array}
$$

Here $\Omega$ is an open bounded Lipschitz polyhedron in $\mathbb{R}^{3}, \partial \Omega$ its boundary, $\mu$ and $\varepsilon$ are the usual electromagnetic tensors; the hypotheses on them are quite general, we shall make them precise later on, but we stress that our assumptions basically allow most practical situations to be set into this framework (see Rem. 1).

A finite element approximation of problem (1) has been analyzed by Monk [16]. In that paper it has been observed that if $\sigma$ is not zero then problem (1) is elliptic. On the other hand the case $\sigma=0$ is more difficult to handle: in particular the problem is not solvable for any value of $\omega$. When $\sigma=0$, it is clear that, if $\omega^{2}$ equals an interior Maxwell eigenvalue, then problem (1) is not well-posed.

We shall consider the finite element approximation of (1) with $\sigma=0$. The divergence constraint is enforced via a Lagrange multiplier according to the ideas of Kikuchi [14]. The existence and uniqueness of the continuous and discrete solutions (under standard conditions) and the convergence of the approximating solution towards the exact one follows easily from the results proved in this paper, together with the theory presented in [8].

Analogous results have been proven in [16] under stronger regularity assumptions (quasiuniform mesh and $H^{1}$ regularity). Also $[11,17]$ deal with the time-harmonic system (1). All these results, however, use an argument which relies on an inverse inequality, so that a quasiuniformity of the mesh is assumed.

We point out that in the present work we do not aim on giving a short convergence proof for the approximation of the time-harmonic Maxwell equations. To this aim, one could easily prove the convergence by putting together the results of [11], where the main Theorem 6 relies on an estimate for the approximation of the Maxwell eigenvalues and those of $[3,4,6]$, where such estimate is proved. The main result of this paper consists in proving the convergence of $T_{h}$ to $T$, where we are using the notation of Section 2 . We observe that then one can obtain the convergence result for the approximation of the time-harmonic Maxwell equations also by using different techniques.

The arguments of our proofs hold for various edge element families, which share the same abstract properties as the Nédélec ones $[4,18]$. For instance, the $h p$-adaptive finite element families presented in [10] fit within our analysis as well.

The outline of the paper is the following. In the next section we present the continuous and discrete operators we shall deal with, for which we will prove a suitable convergence in Section 3. In Section 4 the application of our results to the time-harmonic Maxwell equations is presented. Finally, Section 5 recalls the known families of edge elements to which our results apply.

\section{The MAXwell RESOlVEnt OPERATOR AND ITS DISCRETIZATION}

Let $\Omega$ be an open bounded Lipschitz polyhedron in $\mathbb{R}^{3}, \partial \Omega$ its boundary and $\underline{n}$ the outward oriented normal unit vector. As usual we denote by $\mu, \varepsilon: \Omega \rightarrow \mathbb{R}_{s y m}^{3 \times 3}$ the magnetic permeability and the electric permittivity, respectively. These tensors are elliptic in the sense that

$$
\sum_{i, j=1}^{3} \mu_{i, j}(\underline{x}) \xi_{i} \xi_{j} \geq \mu_{0}|\underline{\xi}|^{2} \quad \sum_{i, j=1}^{3} \varepsilon_{i, j}(\underline{x}) \xi_{i} \xi_{j} \geq \varepsilon_{0}|\underline{\xi}|^{2}
$$

for any real vector $\underline{\xi}=\left(\xi_{1}, \xi_{2}, \xi_{3}\right)$ and for almost any $\underline{x} \in \Omega$ with $\mu_{0}$ and $\varepsilon_{0}$ positive constants. Moreover, they are symmetric and $\bar{b}$ ounded, that is for $i, j=1,2,3 \mu_{i, j}=\mu_{j, i} \in L^{\infty}(\Omega)$ and $\varepsilon_{i, j}=\varepsilon_{j, i} \in L^{\infty}(\Omega)$.

Some regularity assumptions on these coefficients will be made in order to get the inclusion (6). We shall comment on these hypotheses later on. 
To fix the notation, we recall some commonly used functional spaces. Note that, since we will restrict our analysis to the choice $\sigma=0$, all our functions will be real-valued.

$$
\begin{array}{ll}
L^{2}(\Omega) & =\left\{v: \Omega \rightarrow \mathbb{R} \mid \int_{\Omega} v^{2}<+\infty\right\} \\
L_{0}^{2}(\Omega) & =\left\{v \in L^{2}(\Omega): \int_{\Omega} v=0\right\} \\
H^{1}(\Omega) & =\left\{v \in L^{2}(\Omega) \mid \operatorname{grad} v \in L^{2}(\Omega)^{3}\right\} \\
H_{0}^{1}(\Omega) & =\left\{v \in H^{1}(\Omega) \mid v=0 \text { on } \partial \Omega\right\} \\
H(\operatorname{curl} ; \Omega) & =\left\{\underline{v} \in L^{2}(\Omega)^{3} \mid \operatorname{curl} \underline{v} \in L^{2}(\Omega)^{3}\right\} \\
H_{0}(\operatorname{curl} ; \Omega) & =\{\underline{v} \in H(\operatorname{curl} ; \Omega) \mid \underline{v} \times \underline{n}=0 \text { on } \partial \Omega\} \\
H(\operatorname{curl} ; \Omega ; \gamma) & =\left\{\underline{v} \in L^{2}(\Omega)^{3} \mid \operatorname{curl}(\gamma \underline{v}) \in L^{2}(\Omega)^{3}\right\} \\
H(\operatorname{div} ; \Omega) & =\left\{\underline{v} \in L^{2}(\Omega)^{3} \mid \operatorname{div} \underline{v} \in L^{2}(\Omega)\right\} \\
H\left(\operatorname{div}^{0} ; \Omega\right) & =\{\underline{v} \in H(\operatorname{div} ; \Omega) \mid \operatorname{div} \underline{v}=0 \text { in } \Omega\} \\
H\left(\operatorname{div}^{0} ; \Omega ; \gamma\right) & =\left\{\underline{v} \in L^{2}(\Omega)^{3} \mid \operatorname{div}(\gamma \underline{v}) \in L^{2}(\Omega)\right\} \\
H\left(\operatorname{div}^{0} ; \Omega ; \gamma\right) & =\{\underline{v} \in H(\operatorname{div} ; \Omega ; \gamma) \mid \operatorname{div}(\gamma \underline{v})=0 \text { in } \Omega\} \\
H_{0}\left(\operatorname{div}^{0} ; \Omega ; \gamma\right) & =\left\{\underline{v} \in H\left(\operatorname{div}{ }^{0} ; \Omega ; \gamma\right), \mid(\gamma \underline{v}) \cdot \underline{n}=0 \text { on } \partial \Omega\right\}
\end{array}
$$

The symbol $\gamma$ in (2) generally denotes a tensor-valued function defined in $\Omega$.

Then we consider the resolvent operator associated to the Maxwell equations, that is the linear operator $T \in \mathcal{L}\left(L^{2}(\Omega)^{3} ; L^{2}(\Omega)^{3}\right)$ defined as follows. For all $\underline{g} \in L^{2}(\Omega)^{3}, T \underline{g}=\underline{u} \in L^{2}(\Omega)^{3}$ is the first component of the solution of the following problem:

$$
\begin{aligned}
& \text { find }(\underline{u}, p) \in H_{0}(\operatorname{curl} ; \Omega) \times H_{0}^{1}(\Omega) \text { such that } \\
& \begin{cases}\left(\mu^{-1} \operatorname{curl} \underline{u}, \operatorname{curl} \underline{v}\right)+(\varepsilon \underline{v}, \operatorname{grad} p)=(\varepsilon \underline{g}, \underline{v}) & \forall \underline{v} \in H_{0}(\operatorname{curl} ; \Omega) \\
(\varepsilon \underline{u}, \operatorname{grad} q)=0 & \forall q \in H_{0}^{1}(\Omega) .\end{cases}
\end{aligned}
$$

First of all let us prove the compactness of $T$ from $L^{2}(\Omega)^{3}$ into itself.

Lemma 1. The operator $T \in \mathcal{L}\left(L^{2}(\Omega)^{3} ; L^{2}(\Omega)^{3}\right)$ is compact.

Proof. We consider the following orthogonal decomposition of $\underline{g}$ with respect to the scalar product $(\varepsilon \cdot, \cdot)$ (see Eq. (4.14) in [13])

$$
\underline{g}=\underline{\alpha}+\operatorname{grad} \varphi, \quad \text { with } \underline{\alpha} \in H\left(\operatorname{div}^{0} ; \Omega ; \varepsilon\right), \varphi \in H_{0}^{1}(\Omega) .
$$

Notice that the following characterization of $H\left(\operatorname{div}^{0} ; \Omega ; \varepsilon\right)$ holds true

$$
H\left(\operatorname{div}^{0} ; \Omega ; \varepsilon\right)=\left\{\underline{v} \in L^{2}(\Omega)^{3}:(\varepsilon \underline{v}, \operatorname{grad} q)=0 \forall q \in H_{0}^{1}(\Omega)\right\} .
$$

Then we can split (3) into two subproblems taking in (3) first $\underline{v}=\operatorname{grad} \psi$ with $\psi \in H_{0}^{1}(\Omega)$ and next $\underline{v} \in$ $H\left(\operatorname{div}^{0} ; \Omega ; \varepsilon\right)$

$$
\begin{aligned}
& \text { find } p \in H_{0}^{1}(\Omega) \text { such that } \\
& (\varepsilon \operatorname{grad} \psi, \operatorname{grad} p)=(\varepsilon \operatorname{grad} \varphi, \operatorname{grad} \psi) \quad \forall \psi \in H_{0}^{1}(\Omega) ; \\
& \text { find } \underline{u} \in H_{0}(\operatorname{curl} ; \Omega) \cap H\left(\operatorname{div}^{0} ; \Omega ; \varepsilon\right) \text { such that } \\
& \left(\mu^{-1} \operatorname{curl} \underline{u}, \operatorname{curl} \underline{v}\right)=(\varepsilon \underline{\alpha}, \underline{v}) \quad \forall \underline{v} \in H\left(\operatorname{div}^{0} ; \Omega ; \varepsilon\right) .
\end{aligned}
$$

Thanks to the fact that the bilinear form $\left(\mu^{-1} \operatorname{curl} \underline{u}, \operatorname{curl} \underline{v}\right)$ is symmetric, continuous and coercive on the space $H_{0}(\operatorname{curl} ; \Omega) \cap H\left(\operatorname{div}^{0} ; \Omega ; \varepsilon\right)$ and to the compact embedding of $H_{0}(\operatorname{curl} ; \Omega) \cap H\left(\operatorname{div}^{0} ; \Omega ; \varepsilon\right)$ into $L^{2}(\Omega)^{3}(\operatorname{see}[1,13])$ the operator $T$ is compact and self-adjoint. 
Let $E$ be the space containing all $\underline{u}$ which solve problem (3). We assume that $\Omega, \mu$ and $\varepsilon$ are such that for some $s>1 / 2$ it holds

$$
E \subseteq H^{s}(\Omega)^{3}
$$

This is our main assumption on the coefficients and on the domain. This hypothesis might be restrictive (discontinuous coefficients $\varepsilon$ and $\mu$ are not allowed), but it could be weakened with the arguments of [9]. In addition to that, (6) is the minimal hypothesis for the standard edge element interpolant to be defined [1].

Remark 1. Recent results by Schöberl [20] show that it is possible to define a Clément-like interpolant for lowest order edge elements on tetrahedra. Moreover, the operator can be set into the standard framework of the commuting diagram (see (15) and the discussion below). By making use of this operator, the results of [3] extend in a natural way to the more general situation $E \subseteq H^{s}(\Omega)^{3}$, with $s>0$ and all the results presented in this paper could be stated in this more general setting.

We now define the finite element spaces we shall work with and the discrete operator $T_{h}$.

Let $\mathcal{T}_{h}$ be a triangulation of $\Omega$. For simplicity we consider a mesh of tetrahedra and Nédélec elements of the first type [18]. Nevertheless, our results apply to a large part of the known families of edge elements. A list of the available choices is given in Section 5 .

Let $k \geq 0$ be a fixed integer number, we define the following finite element spaces:

$$
\begin{aligned}
& E_{h}=\left\{\underline{v} \in H_{0}(\operatorname{curl} ; \Omega):\left.\underline{v}\right|_{K} \in \mathcal{E}_{k}(K) \forall K \in \mathcal{T}_{h}\right\} \\
& Q_{h}=\left\{q \in H_{0}^{1}(\Omega):\left.q\right|_{K} \in \mathcal{P}_{k+1}(K) \forall K \in \mathcal{T}_{h}\right\}
\end{aligned}
$$

where $\mathcal{P}_{k}(K)$ is the set of the restrictions to $K$ of polynomials of degree less than or equal to $k$ and the elements of $\mathcal{E}_{k}(K)$ have the form $\underline{p}(\underline{x})+\underline{r}(\underline{x})$ with $\underline{p} \in \mathcal{P}_{k}(K)^{3}$ and $\underline{r} \in \mathcal{P}_{k+1}(K)^{3}$ such that $\underline{r} \cdot \underline{x} \equiv 0$.

We define the discrete operator $T_{h} \in \overline{\mathcal{L}}\left(L^{2}(\Omega)^{3} ; E_{h}\right)$ as follows. For all $\underline{g} \in L^{2}(\Omega)^{3}, T_{h} g=\underline{u}_{h} \in E_{h}$ is the first component of the solution of the following problem:

$$
\begin{aligned}
& \text { find }\left(\underline{u}_{h}, p_{h}\right) \in E_{h} \times Q_{h} \text { such that } \\
& \begin{cases}\left(\mu^{-1} \operatorname{curl} \underline{u} \underline{u}_{h}, \operatorname{curl} \underline{v}\right)+\left(\varepsilon \underline{v}, \operatorname{grad} p_{h}\right)=(\varepsilon \underline{g}, \underline{v}) & \forall \underline{v} \in E_{h} \\
\left(\varepsilon \underline{u}_{h}, \operatorname{grad} q\right)=0 & \forall q \in Q_{h} .\end{cases}
\end{aligned}
$$

It is well-known $[1,3,14]$ that problem $(8)$ has a unique solution which is stable in the sense that

$$
\left\|\underline{u}_{h}\right\|_{0}+\left\|\operatorname{curl} \underline{u}_{h}\right\|_{0}+\left\|p_{h}\right\|_{1} \leq C\|\underline{g}\|_{0}
$$

\section{Convergence Results}

The aim of this section is to prove the uniform convergence of $T_{h}$ to $T$. We need to show that the following property holds true: there exists $\rho(h)$, tending to zero as $h$ goes to zero, such that for any $\underline{g} \in L^{2}(\Omega)^{3}$ one has

$$
\left\|T \underline{g}-T_{h} \underline{g}\right\|_{0} \leq \rho(h)\|\underline{g}\|_{0} .
$$

In the case of $\underline{g}$ belonging to $H\left(\operatorname{div}^{0} ; \Omega ; \varepsilon\right)$ this result is a consequence of Theorem 1 in [3], as it will be shown below for sake of completeness.

Let us recall some results on finite element spaces $E_{h}$ and $Q_{h}$ which will be useful to prove the convergence result (10) (see, e.g., $[18,19]$ ). First of all, we introduce the interpolation operators in $Q_{h}$ and $E_{h}$ and their approximation properties.

We denote by $\Pi_{h}^{Q}$ the usual nodal interpolation operator onto $Q_{h}$ : for each function $q \in H_{0}^{1}(\Omega)$ smooth enough, $\Pi_{h}^{Q} q \in Q_{h}$ is obtained using the values at the nodes. The following interpolation error estimate is 
well-known

$$
\left\|q-\Pi_{h}^{Q} q\right\|_{1} \leq C h^{r-1}\|q\|_{r} \quad 1 \leq r \leq k+1
$$

Let $\Pi_{h}^{E}$ be the standard interpolation operator which to each function $\underline{v}$ of $H^{s}(\Omega)^{3}$ associates the element $\Pi_{h}^{E} \underline{v} \in E_{h}$ using the following degrees of freedom: the moments of the tangential component of degree at most $k$ on each edge of $K$, the moments of the tangential component of degree at most $k-1(k>0)$ on each face of $K$ and the moments of degree at most $k-2(k>1)$ on the tetrahedron $K$.

The following approximation properties hold true for all $\underline{v} \in E$ (see [1])

$$
\begin{aligned}
& \left\|\underline{v}-\Pi_{h}^{E} \underline{v}\right\|_{0} \leq C h^{s}\left(|\underline{v}|_{s}+|\operatorname{curl} \underline{v}|_{s}\right) \quad 1 / 2<s \leq k+1 \\
& \left\|\operatorname{curl} \underline{v}-\operatorname{curl} \Pi_{h}^{E} \underline{v}\right\|_{0} \leq C h^{t}|\operatorname{curl} \underline{v}|_{t} 0<t \leq k+1
\end{aligned}
$$

The first of (12) can be improved when curl $\underline{v}$ belongs to $F_{h}$ in the following way:

$$
\left\|\underline{v}-\Pi_{h}^{E} \underline{v}\right\|_{0} \leq C h^{s}|\underline{v}|_{s} \quad 1 / 2<s \leq k+1
$$

A property which will play an essential role in the proof of the convergence (10) is the commuting diagram property. Let us introduce the Raviart-Thomas face discretization of $H_{0}(\operatorname{div} ; \Omega)$. We denote by $F_{h}$ the $k$-th order Raviart-Thomas space corresponding to $E_{h}$, that is

$$
F_{h}=\left\{\underline{v} \in H_{0}(\operatorname{div} ; \Omega):\left.\underline{v}\right|_{K} \in \mathcal{F}_{k}(K) \forall K \in \mathcal{T}_{h}\right\}
$$

where $\mathcal{F}_{k}(K)$ contains the vector fields of the form $\underline{p}(\underline{x})+p(\underline{x}) \underline{x}$, with $\underline{p} \in \mathcal{P}_{k}(K)^{3}$ and $p \in \mathcal{P}_{k}(K)$. The standard face interpolant operator $\Pi_{h}^{F}$ associates to any smooth enough vectorfield $\underline{v}$ a discrete element $\Pi_{h}^{F} \underline{v} \in F_{h}$ by using the following degrees of freedom: the moments of the normal component of degree at most $k$ on each face of $K$ and the moments of degree at most $k-1(k>0)$ on the tetrahedron $K$.

At the end, let $S_{h}$ be the finite dimensional subspace of $L^{2}(\Omega)$ containing discontinuous piecewise polynomials of degree not greater than $k$. The degrees of freedom used in order to define the interpolant operator $\Pi_{h}^{S}$ are the moments of degree at most $k$ on the tetrahedron $K$.

Then the commuting diagram property can be pictured in the following graph:

$$
\begin{aligned}
& 0 \rightarrow Q \quad \stackrel{\text { grad }}{\longrightarrow} E \quad \stackrel{\text { curl }}{\longrightarrow} F \quad \stackrel{\text { div }}{\longrightarrow} S / \mathbb{R} \quad \rightarrow 0 \\
& \downarrow \Pi_{h}^{Q} \quad \downarrow \Pi_{h}^{E} \quad \downarrow \Pi_{h}^{F} \quad \downarrow \Pi_{h}^{S} \\
& 0 \rightarrow Q_{h} \quad \stackrel{\text { grad }}{\longrightarrow} E_{h} \quad \stackrel{\text { curl }}{\longrightarrow} F_{h} \quad \stackrel{\text { div }}{\longrightarrow} S_{h} / \mathbb{R} \rightarrow 0
\end{aligned}
$$

In the above diagram, the spaces $Q, F, S$ are suitable smooth dense subspaces of $H_{0}^{1}(\Omega), H_{0}(\operatorname{div} ; \Omega)$ and $L^{2}(\Omega)$, respectively.

Let us now prove the uniform convergence (10). First of all, we observe that (10) holds true when $\underline{g} \in$ $H\left(\operatorname{div}^{0} ; \Omega ; \varepsilon\right)$, as it can be deduced from Theorem 1 of $[3]$ as follows.

Let us consider the following mixed problem

$$
\begin{aligned}
& \text { given } \underline{G} \in H_{0}\left(\operatorname{div}^{0} ; \Omega ; \mu^{1 / 2}\right), \text { find }(\underline{\sigma}, \underline{w}) \in H_{0}(\operatorname{curl} ; \Omega) \times H_{0}\left(\operatorname{div}^{0} ; \Omega ; \mu^{1 / 2}\right) \text { such that } \\
& \left\{\begin{array}{cc}
(\varepsilon \underline{\sigma}, \underline{\tau})+\left(\mu^{-1 / 2} \operatorname{curl} \underline{\tau}, \underline{w}\right)=0 \quad \forall \underline{\tau} \in H_{0}(\operatorname{curl} ; \Omega) \\
\left(\mu^{-1 / 2} \operatorname{curl} \underline{\sigma}, \underline{z}\right)=(\underline{G}, \underline{z}) \quad \forall \underline{z} \in H_{0}\left(\operatorname{div}^{0} ; \Omega ; \mu^{1 / 2}\right)
\end{array}\right.
\end{aligned}
$$


and the corresponding approximation

$$
\begin{aligned}
& \text { given } \underline{G} \in H_{0}\left(\operatorname{div}^{0} ; \Omega ; \mu^{1 / 2}\right), \text { find }\left(\underline{\sigma}_{h}, \underline{w}_{h}\right) \in E_{h} \times W_{h} \text { such that } \\
& \left\{\begin{array}{lc}
\left(\underline{\sigma}_{h}, \underline{\tau}\right)+\left(\mu^{-1 / 2} \operatorname{curl} \underline{\tau}, \underline{w}_{h}\right)=0 & \forall \underline{\tau} \in E_{h} \\
\left(\mu^{-1 / 2} \operatorname{curl} \underline{\sigma}_{h}, \underline{z}\right)=(\underline{G}, \underline{z}) & \forall \underline{z} \in W_{h} .
\end{array}\right.
\end{aligned}
$$

Here $W_{h}$ is the finite element subspace of $H_{0}\left(\operatorname{div}^{0} ; \Omega ; \mu^{1 / 2}\right)$ constructed using the face elements; more precisely it can be characterized as $W_{h}=\mu^{-1 / 2}\left(F_{h} \cap H\left(\operatorname{div}^{0} ; \Omega\right)\right)=\mu^{-1 / 2} \operatorname{curl} E_{h}$.

Notice that if the data $\underline{g}$ in (3) and $\underline{G}$ in (16) are related by

$$
\varepsilon \underline{g}=\operatorname{curl}\left(\mu^{-1 / 2} \underline{G}\right),
$$

then problems (3) and (16) ( (8) and (17), respectively) are equivalent in the sense stated by the following proposition. We remark that, if $\underline{G} \in H_{0}\left(\operatorname{div}^{0} ; \Omega ; \mu^{1 / 2}\right) \cap H\left(\operatorname{curl} ; \Omega ; \mu^{-1 / 2}\right)$, then $\varepsilon^{-1} \operatorname{curl}\left(\mu^{-1 / 2} \underline{G}\right)$ belongs to $H\left(\operatorname{div}^{0} ; \Omega ; \varepsilon\right)$. On the other hand, given $\underline{g} \in H\left(\operatorname{div}^{0} ; \Omega ; \varepsilon\right)$, then there exists $\underline{G} \in H_{0}\left(\operatorname{div}^{0} ; \Omega ; \mu^{1 / 2}\right) \cap$ $H\left(\operatorname{curl} ; \Omega ; \mu^{-1 / 2}\right)$ such that (18) holds true. This last claim can be proved, for instance, by considering the following auxiliary mixed problem

$$
\begin{aligned}
& \text { find } \underline{G} \in H\left(\operatorname{curl} ; \Omega ; \mu^{-1 / 2}\right) \text { and } r \in H^{1}(\Omega) \cap L_{0}^{2}(\Omega) \text { such that: } \\
& \left\{\begin{array}{l}
\left(\operatorname{curl} \mu^{-1 / 2} \underline{G}, \operatorname{curl} \mu^{-1 / 2} \underline{\gamma}\right)+\left(\mu^{1 / 2} \underline{\gamma}, \operatorname{grad} r\right)=\left(\varepsilon \underline{g}, \operatorname{curl} \mu^{-1 / 2} \underline{\gamma}\right) \begin{array}{l}
\forall \underline{\gamma} \in H\left(\operatorname{curl} ; \Omega ; \mu^{-1 / 2}\right) \\
\forall s \in H^{1}(\Omega) \cap L_{0}^{2}(\Omega) .
\end{array}
\end{array}\right.
\end{aligned}
$$

The well-posedness of problem (19) can be shown by using the orthogonal decomposition

$$
\underline{G}=\mu^{-1 / 2} \operatorname{curl} \underline{\varphi}+\mu^{1 / 2} \operatorname{grad} \psi
$$

with $\underline{\varphi} \in H_{0}(\operatorname{curl} ; \Omega) \cap H\left(\operatorname{div}^{0} ; \Omega\right)$ and $\psi \in H^{1}(\Omega) \cap L_{0}^{2}(\Omega)$ (see Prop. 6.1 of [13]).

Proposition 1. Let $\underline{g} \in H\left(\operatorname{div}^{0} ; \Omega ; \varepsilon\right)$ and $\underline{G} \in H_{0}\left(\operatorname{div}^{0} ; \Omega ; \mu^{1 / 2}\right)$ be such that relation (18) is satisfied. Let $(\underline{u}, p)$ be the solution of (3), then $\underline{u}$ is the first component of the solution of (16). Conversely, if ( $\underline{\sigma}, \underline{w})$ is a solution of (16), then $\underline{\sigma}$ is the first component of the solution of (3) with datum $\underline{g} \in H\left(\operatorname{div}^{0} ; \Omega ; \varepsilon\right)$.

Let $\left(\underline{u}_{h}, p_{h}\right)$ be the solution of (8), then $\underline{u}_{h}$ is the first component of the solution of (17). Conversely, if $\left(\underline{\sigma}_{h}, \underline{w}_{h}\right)$ is a solution of (17), then $\underline{\sigma}_{h}$ is the first component of the solution of (8).

Proof. Since $\operatorname{grad} p$ belongs to $H_{0}(\operatorname{curl} ; \Omega)$, we can take $\underline{v}=\operatorname{grad} p$ in the first equation of (3) and we obtain that $p=0$. Hence, using also relation (18), we can write

$$
\left(\mu^{-1 / 2} \operatorname{curl} \underline{u}, \mu^{-1 / 2} \operatorname{curl} \underline{v}\right)=\left(\operatorname{curl}\left(\mu^{-1 / 2} \underline{G}\right), \underline{v}\right) \quad \forall \underline{v} \in H_{0}(\operatorname{curl} ; \Omega) .
$$

Since $\mu^{-1 / 2} \operatorname{curl}\left(H_{0}(\operatorname{curl} ; \Omega)\right)=H_{0}\left(\operatorname{div}^{0} ; \Omega ; \mu^{1 / 2}\right)$ an integration by parts gives

$$
\left(\mu^{-1 / 2} \operatorname{curl} \underline{u}, \underline{z}\right)=(\underline{G}, \underline{z}) \quad \forall \underline{z} \in H_{0}\left(\operatorname{div}^{0} ; \Omega ; \mu^{1 / 2}\right) .
$$

Now we need to show that there exists $\underline{w} \in H_{0}\left(\operatorname{div}^{0} ; \Omega ; \mu^{1 / 2}\right)$ such that the first equation in (16) is satisfied with $\underline{\sigma}=\underline{u}$, namely

$$
\left(\underline{w}, \mu^{-1 / 2} \operatorname{curl} \underline{\tau}\right)=-(\varepsilon \underline{u}, \underline{\tau}) \quad \forall \underline{\tau} \in H_{0}(\operatorname{curl} ; \Omega) .
$$

This last equation is clearly solvable, since the compatibility for the right-hand side reads $(\varepsilon \underline{u}$, $\operatorname{grad} q)=0$ for any $q \in H_{0}^{1}(\Omega)$, which is exactly the second equation in (3). 
Conversely, using again the fact that $\mu^{-1 / 2} \operatorname{curl}\left(H_{0}(\operatorname{curl} ; \Omega)\right)=H_{0}\left(\operatorname{div}^{0} ; \Omega ; \mu^{1 / 2}\right)$ we have that the second equation of (16) implies the first equation of (3). Then taking $\underline{z}=\operatorname{grad} q$ for all $q \in H_{0}^{1}(\Omega)$ in the first equation of (16) gives the second equation of (3).

The proof of the equivalence between problems (8) and (17) is similar, thanks to the commuting diagram property (15) and the definition of $W_{h}$.

A consequence of the main result in [3] is the following error estimate for the solutions of (3) and (8):

Proposition 2. Let us assume that (6) is fulfilled and that $\underline{g} \in H\left(\operatorname{div}^{0} ; \Omega ; \varepsilon\right)$. Let $\underline{u} \in H_{0}(\operatorname{curl} ; \Omega)$ and $\underline{u}_{h} \in E_{h}$ be the first components of the solutions of (3) and (8), respectively. Then

$$
\left\|\underline{u}-\underline{u}_{h}\right\|_{0} \leq C h^{s}\|\underline{g}\|_{0} .
$$

Proof. From Prop. 1, we have that $\underline{u}=\underline{\sigma}$ and $\underline{u}_{h}=\underline{\sigma}_{h}$, where $\underline{\sigma}$ and $\underline{\sigma}_{h}$ are the first components of the solutions of problems (16) and (17), respectively, with datum $\underline{G} \in H_{0}\left(\operatorname{div}^{0} ; \Omega ; \mu^{1 / 2}\right) \cap H\left(\operatorname{curl} ; \Omega ; \mu^{-1 / 2}\right)$ satisfying (18).

A direct consequence of Theorem 1 in [3] is that the following estimate holds true

$$
\left\|\underline{u}-\underline{u}_{h}\right\|_{0}=\left\|\underline{\sigma}-\underline{\sigma}_{h}\right\|_{0} \leq h^{s}\|\underline{G}\|_{0} .
$$

Thanks to (18) and to the fact that $\underline{G} \in H_{0}\left(\operatorname{div}^{0} ; \Omega ; \mu^{1 / 2}\right) \cap H\left(\operatorname{curl} ; \Omega ; \mu^{-1 / 2}\right)$, we have that

$$
\|\underline{G}\|_{0} \leq C\left\|\operatorname{curl}\left(\mu^{-1 / 2} \underline{G}\right)\right\|_{0} \leq C\|\underline{g}\|_{0} .
$$

Inserting the last inequality in (24) gives (23).

Let us now go back to the main result of this section, that is we need show that (10) holds true for any $\underline{g} \in L^{2}(\Omega)^{3}$. We first observe that it suffices to prove the same property for any discrete function $\underline{g}_{h} \in E_{h}$, namely

$$
\left\|T \underline{g}_{h}-T_{h} \underline{g}_{h}\right\|_{0} \leq C h^{s}\left\|\underline{g}_{h}\right\|_{0} .
$$

This is a consequence of the results of [12] and of Theorem 5.1 in [5]. Indeed, the discretely uniform convergence (25), together with suitable approximation properties, implies the good convergence of the eigenmodes of $T_{h}$ towards those of $T$. On the other hand, the good convergence of the eigenmodes is equivalent to (10), since $T$ is compact and self-adjoint.

More precisely, let $\nu \neq 0$ be an isolated eigenvalue of $T$ with finite algebraic multiplicity $m$, then there exists a closed ball $B$, centered at $\nu$, which does not contain any other element of the spectrum of $T$. Let $\nu_{1, h}, \cdots, \nu_{m}(h), h$ be the eigenvalues of $T_{h}$ contained in $B$ and repeated according to their algebraic multiplicities. In Section 2 of [12] it has been proved that (25) and (12), (13) imply

$$
\begin{array}{ll}
m(h)=m & \text { for } h \text { small enough, } \\
\lim _{h \rightarrow 0} \nu_{i, h}=\nu & \text { for } i=1, \cdots, m, \\
\lim _{h \rightarrow 0} \hat{\delta}\left(X, X_{h}\right)=0, &
\end{array}
$$

where $X$ and $X_{h}$ denote the eigenspaces associated to $\nu$ and $\nu_{1, h}, \cdots, \nu_{m, h}$, respectively, and $\hat{\delta}\left(X, X_{h}\right)$ denotes the gap between $X$ and $X_{h}$ as closed subspaces of $L^{2}(\Omega)^{3}$.

Then, since the operator $T$ is self-adjoint and compact (see [5]), we obtain as a consequence of (26) the uniform convergence of $T_{h}$ to $T$, that is

$$
\lim _{h \rightarrow 0}\left\|T-T_{h}\right\|_{\mathcal{L}\left(L^{2}(\Omega)^{3} ; L^{2}(\Omega)^{3}\right)}=0 .
$$


The following lemma aims to prove the convergence (25)

Lemma 2. There exists $C$ such that for all $\underline{g}_{h} \in E_{h}$ (25) holds true.

Proof. Since $\operatorname{grad}\left(Q_{h}\right)$ is a subspace of $E_{h}$, we can split $\underline{g}_{h} \in E_{h}$, by a discrete Helmholtz decomposition, as follows:

$$
\underline{g}_{h}=\underline{\alpha}_{h}+\operatorname{grad} \varphi_{h} \quad \text { with } \underline{\alpha}_{h} \in E_{h}, \varphi_{h} \in Q_{h} \text { and }\left(\varepsilon \underline{\alpha}_{h}, \operatorname{grad} q\right)=0 \quad \forall q \in Q_{h} .
$$

Thanks to the linearity of the operators $T$ and $T_{h}$ we have

$$
\begin{aligned}
& T \underline{g}_{h}=\underline{u}_{\alpha}+\underline{u}_{\varphi} \text { with } \underline{u}_{\alpha}, \underline{u}_{\varphi} \in H_{0}(\operatorname{curl} ; \Omega) \\
& T_{h} \underline{g}_{h}=\underline{u}_{\alpha, h}+\underline{u}_{\varphi, h} \text { with } \underline{u}_{\alpha, h}, \underline{u}_{\varphi, h} \in E_{h}
\end{aligned}
$$

where $\underline{u}_{\alpha}=T \underline{\alpha}_{h}$ and $\underline{u}_{\varphi}=T \operatorname{grad} \varphi_{h}$ are the solutions of (3) when $\underline{g}=\underline{\alpha}_{h}$ and $\underline{g}=\operatorname{grad} \varphi_{h}$, respectively. In a similar way $\underline{u}_{\alpha, h}=T_{h} \underline{\alpha}_{h}$ and $\underline{u}_{\varphi, h}=T_{h} \operatorname{grad} \varphi_{h}$ are the solutions of (8).

It is easy to check that $\underline{u}_{\varphi}=\underline{u}_{\varphi, h}=0$. For example let us prove $\underline{u}_{\varphi, h}=0$. In (8) with $\underline{g}=\operatorname{grad} \varphi_{h}$ we take $\underline{v}=\underline{u}_{\varphi, h}$, then we have

$$
\begin{aligned}
& \left(\mu^{-1} \operatorname{curl} \underline{u}_{\varphi, h}, \operatorname{curl} \underline{u}_{\varphi, h}\right)=\left(\varepsilon \operatorname{grad} \varphi_{h}, \underline{u}_{\varphi, h}\right) \\
& \left(\varepsilon \underline{u}_{\varphi, h}, \operatorname{grad} q\right)=0 \quad \forall q \in Q_{h} .
\end{aligned}
$$

Then curl $\underline{u}_{\varphi, h}=0$ and there exists $\chi_{h} \in Q_{h}$ such that $\underline{u}_{\varphi, h}=\operatorname{grad} \chi_{h}$ (see [18]). The second equation in (30) with $q=\chi_{h}$ gives $\underline{u}_{\varphi, h}=0$.

Hence (25) is proved if we estimate $\left\|\underline{u}_{\alpha}-\underline{u}_{\alpha, h}\right\|$.

We express $\underline{\alpha}_{h}$ as follows (see (4) and (5))

$$
\underline{\alpha}_{h}=\underline{\alpha}(h)+\operatorname{grad} \chi(h) \text { with } \underline{\alpha}(h) \in H_{0}(\operatorname{curl} ; \Omega) \cap H\left(\operatorname{div}^{0} ; \Omega ; \varepsilon\right), \chi(h) \in H_{0}^{1}(\Omega) .
$$

Notice that by construction $\underline{\alpha}(h) \in H^{s}(\Omega)^{3}$ due to (6) with the bound

$$
\|\underline{\alpha}(h)\|_{s} \leq C \underline{\alpha}_{h} \|_{0}
$$

Then Proposition 2 implies

$$
\left\|\left(T-T_{h}\right) \underline{\alpha}(h)\right\|_{0} \leq C h^{s}\|\underline{\alpha}(h)\|_{s} \leq C h^{s}\left\|_{h}\right\|_{0} .
$$

It remains to estimate $\left\|\left(T-T_{h}\right) \operatorname{grad} \chi(h)\right\|_{0}$. Due to Lemma 1 and (9) we have

$$
\left\|\left(T-T_{h}\right) \operatorname{grad} \chi(h)\right\|_{0} \leq\|T \operatorname{grad} \chi(h)\|_{0}+\left\|T_{h} \operatorname{grad} \chi(h)\right\|_{0} \leq C\|\operatorname{grad} \chi(h)\|_{0} .
$$

An argument of [2] can be adapted to our situation in order to get a bound for $\|\operatorname{grad} \chi(h)\|_{0}$

$$
\begin{aligned}
& \varepsilon_{0}\|\operatorname{grad} \chi(h)\|_{0}^{2} \leq\left(\varepsilon\left(\underline{\alpha}_{h}-\underline{\alpha}(h)\right), \underline{\alpha}_{h}-\underline{\alpha}(h)\right) \\
& \quad=\left(\varepsilon\left(\underline{\alpha}_{h}-\underline{\alpha}(h)\right), \underline{\alpha}_{h}-\Pi_{h}^{E} \underline{\alpha}(h)\right)+\left(\varepsilon\left(\underline{\alpha}_{h}-\underline{\alpha}(h)\right), \Pi_{h}^{E} \underline{\alpha}(h)-\underline{\alpha}(h)\right) .
\end{aligned}
$$

Let us recall that $F_{h}$ represents the $k$-th order Raviart-Thomas space corresponding to $E_{h}$ (see (14)). From the commuting diagram property (15) we shall make use of the identity

$$
\operatorname{curl} \Pi_{h}^{E} \underline{\alpha}(h)=\Pi_{h}^{F} \operatorname{curl} \underline{\alpha}(h),
$$

where $\Pi_{h}^{F}$ is the standard face interpolant operator. 
With this notation, equation (35) gets

$$
\operatorname{curl} \Pi_{h}^{E} \underline{\alpha}(h)=\Pi_{h}^{F} \operatorname{curl} \underline{\alpha}(h)=\Pi_{h}^{F} \operatorname{curl} \underline{\alpha}_{h}=\operatorname{curl} \underline{\alpha}_{h},
$$

where the last equality is due to the fact that $\operatorname{curl}\left(E_{h}\right)$ is contained in $F_{h}$. Therefore

$$
\operatorname{curl}\left(\underline{\alpha}_{h}-\Pi_{h}^{E} \underline{\alpha}(h)\right)=0 .
$$

Hence there exists $\xi_{h} \in Q_{h}$ such that

$$
\underline{\alpha}_{h}-\Pi_{h}^{E} \underline{\alpha}(h)=\operatorname{grad} \xi_{h} .
$$

Since $\underline{\alpha}(h)$ belongs to $H\left(\operatorname{div}^{0} ; \Omega ; \varepsilon\right)$, then there exists $\underline{\beta}$ such that

$$
\varepsilon \underline{\alpha}(h)=\operatorname{curl} \underline{\beta}
$$

and, putting together (28), (37) and (39), it results

$$
\left(\varepsilon\left(\underline{\alpha}_{h}-\underline{\alpha}(h)\right), \underline{\alpha}_{h}-\Pi_{h}^{E} \underline{\alpha}(h)\right)=-\left(\operatorname{curl} \underline{\beta}, \operatorname{grad} \xi_{h}\right)=-\int_{\partial \Omega} \underline{\beta} \cdot \operatorname{grad} \xi_{h} \times \underline{n}=0 .
$$

We use the last equality in (35) together with (12), (13) and the a priori estimate (32) and we get

$$
\begin{aligned}
\|\operatorname{grad} \chi(h)\|_{0}^{2} & \leq C\left\|\underline{\alpha}_{h}-\underline{\alpha}(h)\right\|_{0}\left\|\Pi_{h}^{E} \underline{\alpha}(h)-\underline{\alpha}(h)\right\|_{0} \\
& \leq C\|\operatorname{grad} \chi(h)\|_{0} h^{s}\|\underline{\alpha}(h)\|_{s} \\
& \leq C\|\operatorname{grad} \chi(h)\|_{0} h^{s}\left\|\underline{\alpha}_{h}\right\|_{0} .
\end{aligned}
$$

The inequalities (33), (34) and (40), together with (28), give

$$
\left\|\left(T-T_{h}\right) \underline{g}_{h}\right\|_{0}=\left\|\underline{u}_{\alpha}-\underline{u}_{\alpha, h}\right\|_{0} \leq C h^{s}\left\|_{h}\right\|_{0} \leq C h^{s}\left\|\underline{g}_{h}\right\|_{0}
$$

which concludes the proof of the lemma.

\section{Application to the time-harmonic Maxwell equations}

Let us consider the time-harmonic Maxwell equations (1) in the case $\sigma=0$. Given $\underline{f} \in H\left(\operatorname{div}^{0} ; \Omega\right)$ and $\omega \in \mathbb{R}$ our problem is to find $\underline{u}: \Omega \rightarrow \mathbb{R}^{3}$ such that

$$
\begin{array}{ll}
\operatorname{curl}\left(\mu^{-1} \operatorname{curl} \underline{u}\right)-\omega^{2} \varepsilon \underline{u}=\underline{f} & \text { in } \Omega, \\
\operatorname{div}(\varepsilon \underline{u})=0 & \text { in } \Omega, \\
\underline{u} \times \underline{n}=0 & \text { on } \partial \Omega .
\end{array}
$$

A variational formulation can be obtained by using a Lagrange multiplier technique to enforce the divergence free constraint as proposed by Kikuchi [14].

$$
\begin{aligned}
& \text { Find }(\underline{u}, p) \in H_{0}(\operatorname{curl} ; \Omega) \times H_{0}^{1}(\Omega) \text { such that } \\
& \left\{\begin{array}{l}
\left(\mu^{-1} \operatorname{curl} \underline{u}, \operatorname{curl} \underline{v}\right)-\omega^{2}(\varepsilon \underline{u}, \underline{v})+(\varepsilon \underline{v}, \operatorname{grad} p)=(\underline{f}, \underline{v}) \begin{array}{r}
\forall \underline{v} \in H_{0}(\operatorname{curl} ; \Omega) \\
\forall q \in H_{0}^{1}(\Omega) .
\end{array}
\end{array}\right.
\end{aligned}
$$

It is not difficult to see that a solution to (42) solves also (43) with $p=0$ and that if (43) has a regular solution $(\underline{u}, p)$ then $\underline{u}$ fulfills equation $(42)$. 
A finite element approximation of problem (43) is obtained by considering a sequence of finite dimensional subspaces $E_{h} \subset H_{0}(\operatorname{curl} ; \Omega)$ and $Q_{h} \subset H_{0}^{1}(\Omega)$ (see Sect. 3 for the case of Nédélec elements of the first type [18]). The discrete problem reads as follows:

$$
\begin{aligned}
& \text { find }\left(\underline{u}_{h}, p_{h}\right) \in E_{h} \times Q_{h} \text { such that } \\
& \left\{\begin{array}{l}
\left(\mu^{-1} \operatorname{curl} \underline{u}_{h}, \operatorname{curl} \underline{v}\right)-\omega^{2}\left(\varepsilon \underline{u}_{h}, \underline{v}\right)+\left(\varepsilon \underline{v}, \operatorname{grad} p_{h}\right)=(\underline{f}, \underline{v}) \begin{array}{l}
\forall \underline{v} \in E_{h} \\
\forall q \in Q_{h} .
\end{array} \\
\left(\varepsilon \underline{u}_{h}, \operatorname{grad} q\right)=0
\end{array}\right.
\end{aligned}
$$

As usual when dealing with mixed methods, the convergence properties of the discrete solution $\left(\underline{u}_{h}, p_{h}\right)$ depend on the choice of the spaces $\left(E_{h}, Q_{h}\right)$. In particular we need some compatibility condition, namely the inf-sup condition and the ellipticity in the kernel property (see [7]). These properties are generally satisfied by the finite element spaces we shall consider (see Sects. 3 and 5).

In order to obtain the convergence of the discrete solution of (44) to the continuous one, we shall apply the uniform convergence (27) that we have proved in the previous section. This will be done following an approach to the problem which makes use of a new abstract setting. For our proof we adapt a part of the theory presented in [8] for the approximation of regular branches of nonlinear problems. The solution to the problem (42) will be defined implicitly via a suitable nonlinear equation.

Let us recall the abstract setting introduced in [8] for the analysis of the numerical approximation of nonlinear problems. Let $V$ and $W$ be two Hilbert spaces, $\Lambda \subset \mathbb{R}$ a compact interval, $G: \Lambda \times V \rightarrow W$ a $C^{1}$ mapping and $T \in \mathcal{L}(W ; V)$ a linear continuous mapping. We consider the equation

$$
u+T G(\lambda, u)=0
$$

and we make the following assumptions on the nonlinear problem (45):

[A1] for all $(\lambda, u) \in \Lambda \times V$, the operator $T D_{u} G(\lambda, u) \in \mathcal{L}(V ; V)$ is compact;

[A2] there exists a branch $\{(\lambda, u(\lambda)), \lambda \in \Lambda\}$ of nonsingular solutions of (45), such that $\lambda \rightarrow u(\lambda)$ is a $C^{1}$ function from $\Lambda$ to $V$. Moreover for all $\lambda \in \Lambda u(\lambda) \in Z$ where $Z \subset V$ is an Hilbert space;

[A3] the mapping $(\lambda, u) \in \Lambda \times V \rightarrow D G(\lambda, u) \in \mathcal{L}(\mathbb{R} \times V ; W)$ is Lipschitz continuous on the bounded subsets of $\Lambda \times V$, i.e., there exists a function $L: \mathbb{R}_{+} \times \mathbb{R}_{+} \rightarrow \mathbb{R}_{+}$monotonically increasing with respect to each variable such that for all $\lambda_{1}, \lambda_{2} \in \Lambda$ and all $u_{1}, u_{2} \in V$

$$
\left\|D G\left(\lambda_{1}, u_{1}\right)-D G\left(\lambda_{2}, u_{2}\right)\right\|_{\mathcal{L}(\mathbb{R} \times V ; W)} \leq L\left(\left|\lambda_{1}\right|+\left\|u_{1}\right\|_{V},\left|\lambda_{2}\right|+\left\|u_{2}\right\|_{V}\right)\left(\left|\lambda_{1}-\lambda_{2}\right|+\left\|u_{1}-u_{2}\right\|_{V}\right) .
$$

Let $V_{h}$ be a finite dimensional subspace of $V$ and $T_{h} \in \mathcal{L}\left(W ; V_{h}\right)$ a linear operator with finite range, then the discretization of equation (45) reads:

$$
u_{h}+T_{h} G\left(\lambda, u_{h}\right)=0 .
$$

The finite element space $V_{h}$ and the discrete linear operator $T_{h}$ are assumed to satisfy the following conditions: [D1] there exists a linear operator $\Pi_{h}: Z \rightarrow V_{h}$ such that the function $\Pi_{h} u: \lambda \in \Lambda \rightarrow \Pi_{h} u(\lambda) \in V_{h}$ fulfills

$$
\left\|\Pi_{h} u\left(\lambda_{1}\right)-\Pi_{h} u\left(\lambda_{2}\right)\right\|_{V} \leq C\left|\lambda_{1}-\lambda_{2}\right| \text { for all } \lambda_{1}, \lambda_{2} \in \Lambda
$$

and for some positive real number $r$

$$
\sup _{\lambda \in \Lambda}\left\|u(\lambda)-\Pi_{h} u(\lambda)\right\|_{V} \leq C h^{r},
$$

with $C$ independent of $h$;

[D2] the discrete operator $T_{h}$ converges uniformly to $T$ as $h$ goes to 0 , that is

$$
\lim _{h \rightarrow 0}\left\|T-T_{h}\right\|_{\mathcal{L}(W ; V)}=0 ;
$$


[D3] there exists a positive constant $C$ independent of $h$ such that

$$
\sup _{\lambda \in \Lambda}\left\|\left(T-T_{h}\right) G(\lambda, u(\lambda))\right\|_{V} \leq C h^{r}
$$

The following theorem establishes the existence of a branch $\left\{\left(\lambda, u_{h}(\lambda)\right), \lambda \in \Lambda\right\}$ of solutions of the equation (47) which approximates the branch of nonsingular solutions of (45), see [8] for the details of the proof.

Theorem 1. Assume that hypotheses A1-A3 and D1-D3 hold. Then there exist $h_{0}$ small enough and $K>0$, independent of $\lambda$, such that for all $h<h_{0}$ there is a unique $C^{1}$ mapping $\lambda \rightarrow u_{h}(\lambda) \in V_{h}$ such that for all $\lambda \in \Lambda$

$$
\begin{aligned}
& u_{h}(\lambda)+T_{h} G\left(\lambda, u_{h}(\lambda)\right)=0, \\
& \left\|u(\lambda)-u_{h}(\lambda)\right\|_{V} \leq K h^{r} .
\end{aligned}
$$

We write problem (42) within this framework by means of the following identifications:

$$
\begin{aligned}
& \lambda=\omega^{2} ; \\
& V=W=L^{2}(\Omega)^{3} ; \\
& G(\lambda, \underline{u})=-\lambda \underline{u}-\varepsilon^{-1} \underline{f} ; \\
& Z=E .
\end{aligned}
$$

Then the linear operator $T \in \mathcal{L}\left(L^{2}(\Omega)^{3} ; L^{2}(\Omega)^{3}\right)$ is the resolvent operator associated to the Maxwell equations through (3).

First of all let us check assumptions A1-A3.

The partial derivative $D_{u} G(\lambda, \underline{u}) \in \mathcal{L}\left(\mathbb{R} \times L^{2}(\Omega)^{3} ; L^{2}(\Omega)\right)^{3}$ is given by

$$
D_{u} G(\lambda, \underline{u}) \cdot \underline{v}=-\lambda \underline{v} \quad \forall \lambda \in \Lambda, \underline{u}, \underline{v} \in L^{2}(\Omega)^{3},
$$

hence it is a linear continuous operator from $L^{2}(\Omega)^{3}$ into itself. Then, $T D_{u} G(\lambda, \underline{u})$ is compact thanks to the compactness of $T$ from $L^{2}(\Omega)^{3}$ into itself (see Lem. 1).

The assumption A2 is satisfied if the operator $I+T D_{u} G(\lambda, \underline{u})$ is an isomorphism of $L^{2}(\Omega)^{3}$ into itself. This holds true if $1 / \lambda$ is not an eigenvalue of $T$ due to the Fredholm alternative. Hence $\Lambda$ can be taken to be a closed interval contained in the resolvent set of $T$. Then for all $\lambda \in \Lambda$ the solution $\underline{u}(\lambda)$ belongs to $E$.

Finally, assumption A3 is easily checked since $G$ is linear with respect to each variable.

Let us now consider the discrete problem (44). We take $V_{h}=E_{h}$ and the discrete operator $T_{h} \in \mathcal{L}\left(L^{2}(\Omega)^{3} ; E_{h}\right)$ given by (8).

Let us check that assumptions D1-D3 are satisfied: taking $\Pi_{h}=\Pi_{h}^{E}$, D1 is a consequence of (12), (13); D2 corresponds to the uniform convergence (27). It remains to verify the validity of condition D3. From the definition (53), we have

$$
G(\lambda, \underline{u}(\lambda)) \in H\left(\operatorname{div}^{0} ; \Omega ; \varepsilon\right)
$$

because $\underline{u}(\lambda)$ is a regular solution of $(45)$ and $f \in H\left(\operatorname{div}^{0} ; \Omega\right)$. Then we can apply again Proposition 2 and obtain

$$
\left\|\left(T-T_{h}\right) G(\lambda, \underline{u}(\lambda))\right\|_{0} \leq C h^{s}\|G(\lambda, \underline{u}(\lambda))\|_{0} \quad \forall \lambda \in \Lambda
$$

Thanks to A2, $\Lambda$ is a closed bounded interval in $\mathbb{R}$ and $\lambda \rightarrow \underline{u}(\lambda)$ is a $C^{1}$ function from $\Lambda$ to $L^{2}(\Omega)^{3}$, then we obtain that also D3 holds.

Hence, the hypotheses of Theorem 1 have been proven and we can summarize the results obtained so far in the following theorem. 
Theorem 2. Suppose the regularity assumption (6) is satisfied and let $\Lambda$ be a closed interval contained in the resolvent set of $T$. Then, for $h$ small enough, there exists a unique $C^{1}$ mapping $\lambda \rightarrow \underline{u}_{h}(\lambda)$ from $\Lambda$ to $E_{h}$ such that for all $\lambda \in \Lambda$

$$
\underline{u}_{h}(\lambda)+T_{h} G\left(\lambda, \underline{u}_{h}(\lambda)\right)=0 .
$$

In particular, for all $\omega^{2} \in \Lambda$ there exists a unique solution $\underline{u}_{h}$ of (44) with the following error estimate

$$
\left\|\underline{u}\left(\omega^{2}\right)-\underline{u}_{h}\left(\omega^{2}\right)\right\|_{0} \leq C h^{s} .
$$

\section{FAmilies of edGe ELEMEnts}

In this section we briefly recall the known families of edge elements and generalize the results presented in the previous section. We shall consider the finite element families of Raviart-Thomas-Nédélec $[18,19]$ and the $h p$-adaptive families of Demkowicz-Vardapetyan [21].

For the sake of simplicity, the presentation of this section will be made under the assumptions $\mu=\varepsilon=1$ and $\Omega$ simply connected. These hypotheses allow for an easier and shorter notation.

The hypotheses on the finite element spaces $\left(Q_{h}, E_{h}, F_{h}\right)$, which have been used in order to prove Theorem 2 , can be summarized in the following list:

i) good approximation properties $(c f .(12),(13))$;

ii) commuting diagram property (see (15));

iii) Theorem 1 of [3].

In [4] it has been proved that i) and ii) imply iii). Hence, we concentrate our attention on the commuting diagram property.

\subsection{Tetrahedra}

It is well-known that first and second type Nédélec finite elements on tetrahedra enjoy the commuting diagram property $[18,19]$.

Moreover, the commuting diagram property for the Demkowicz-Vardapetyan elements has been proved in $[10]$.

\subsection{Hexahedra}

The analysis of the general hexahedral case is not so immediate as for the tetrahedral one. In particular, it is apparent that for the second type Nédélec elements the diagram cannot be written.

On the other hand, Demkowicz-Vardapetyan and first type Nédélec elements satisfy the commuting diagram property on meshes of parallelepipeds. On meshes of general hexahedra the result is not so obvious; for instance, for the standard Raviart-Thomas-Nédélec family, the diagram does not commute. However, a suitable definition of the spaces (as suggested in [10]), makes the diagram commute; but good approximation properties for these new spaces are not so obvious.

\section{Conclusions}

In this paper we considered the edge finite element approximation of the Maxwell resolvent operator and we proved the uniform convergence in the $L^{2}(\Omega)^{3}$-norm. The proof of convergence makes use of several interesting features of edge element spaces which are highlighted. Another interesting result which we need in our proof is the one by Descoulx, Nassif and Rappaz [12] which allows us to obtain (10) as a consequence of the weaker property (25). Then we consider the time-harmonic Maxwell equations and the corresponding discretization. The proof of convergence relies on the uniform convergence of the resolvent operator and on the theory of Brezzi-Rappaz-Raviart [8] for the approximation of nonlinear problems. 
In the final section, a brief overview of the known edge element families is included; some problems remain open for meshes of general hexahedra.

\section{REFERENCES}

[1] C. Amrouche, C. Bernardi, M. Dauge and V. Girault, Vector potential in three-dimensional nonsmooth domains. Math Methods Appl. Sci. 21 (1998) 823-864.

[2] A. Bermúdez, R. Durán, A. Muschietti, R. Rodríguez and J. Solomin, Finite element vibration analysis of fluid-solid systems without spurious modes. SIAM J. Numer. Anal. 32 (1995) 1280-1295.

[3] D. Boffi, Fortin operator and discrete compactness for edge elements. Numer. Math. 87 (2000) 229-246.

[4] D. Boffi, A note on the de Rham complex and a discrete compactness property. Appl. Math. Lett. 14 (2001) 33-38.

[5] D. Boffi, F. Brezzi and L. Gastaldi, On the problem of spurious eigenvalues in the approximation of linear elliptic problems in mixed form. Math. Comp. 69 (2000) 121-140.

[6] D. Boffi, P. Fernandes, L. Gastaldi and I. Perugia, Computational models of electromagnetic resonators: analysis of edge element approximation. SIAM J. Numer. Anal. 36 (1998) 1264-1290.

[7] F. Brezzi and M. Fortin, Mixed and hybrid finite element methods. Springer-Verlag, New York (1991).

[8] F. Brezzi, J. Rappaz and P.A. Raviart, Finite dimensional approximation of nonlinear problems. Part i: Branches of nonsingular solutions. Numer. Math. 36 (1980) 1-25.

[9] S. Caorsi, P. Fernandes and M. Raffetto, On the convergence of Galerkin finite element approximations of electromagnetic eigenproblems. SIAM J. Numer. Anal. 38 (2000) 580-607.

[10] L. Demkowicz, P. Monk, L. Vardapetyan and W. Rachowicz, de Rham diagram for $h p$ finite element spaces. Comput. Math. Appl. 39 (2000) 29-38.

[11] L. Demkowicz and L. Vardapetyan, Modeling of electromagnetic absorption/scattering problems using $h p$-adaptive finite elements. Comput. Methods Appl. Mech. Engrg. 152 (1998) 103-124. Symposium on Advances in Computational Mechanics, Vol. 5 (Austin, TX, 1997).

[12] J. Descloux, N. Nassif and J. Rappaz, On spectral approximation. I. The problem of convergence. RAIRO Anal. Numér. 12 (1978) 97-112.

[13] P Fernandes and G. Gilardi, Magnetostatic and electrostatic problems in inhomogeneous anisotropic media with irregular boundary and mixed boundary conditions. Math. Models Methods Appl. Sci. 7 (1997) 957-991.

[14] F. Kikuchi, Mixed and penalty formulations for finite element analysis of an eigenvalue problem in electromagnetism. In Proceedings of the first world congress on computational mechanics (Austin, Tex., 1986), Vol. 64, pages 509-521, 1987.

[15] F. Kikuchi, On a discrete compactness property for the Nédélec finite elements. J. Fac. Sci., Univ. Tokyo, Sect. I A 36 (1989) 479-490.

[16] P. Monk, A finite element method for approximating the time-harmonic Maxwell equations. Numer. Math. 63 (1992) $243-261$.

[17] P. Monk and L. Demkowicz, Discrete compactness and the approximation of Maxwell's equations in $\mathbb{R}^{3}$. Math. Comp. 70 (2001) 507-523.

[18] J.-C. Nédélec, Mixed finite elements in $\mathbb{R}^{3}$. Numer. Math. 35 (1980) 315-341.

[19] J.-C. Nédélec, A new family of mixed finite elements in $\mathbb{R}^{3}$. Numer. Math. 50 (1986) 57-81.

[20] J. Schöberl, Commuting quasi-interpolation operators for mixed finite elements. Preprint ISC-01-10-MATH, Texas A\&M University, 2001.

[21] L. Vardapetyan and L. Demkowicz, hp-adaptive finite elements in electromagnetics. Comput. Methods Appl. Mech. Engrg. 169 (1999) 331-344.

To access this journal online:

www.edpsciences.org 